\title{
Assessment of analytical process performance using the Six Sigma method: A comparison of two biochemistry analyzers
}

\author{
Saniye Basak Oktay, S Sema Nur Ayyildiz \\ Department of Biochemistry, Adiyaman University Training and Research Hospital, Adiyaman, Turkey
}

\begin{abstract}
Objectives: Six Sigma is a method of quality management analysis that integrates accuracy and precision of measurement, error identification, and process improvement. The aim of this study was to evaluate the analytical process performance of routine biochemical tests performed with 2 biochemistry analyzers in our laboratory according to Six Sigma methodology and compare the findings.

Methods: Internal quality control (IQC) data of routine biochemical analytes used for 3 months in 2 Abbott Architect c16000 analyzers (Abbott Diagnostics Inc., Lake Forest, IL, USA) were extracted and the mean, SD, coefficient of variation $\%$, bias $\%$ and sigma values were calculated. The performance of the analytes was classified according to the sigma level: $<3$ demonstrated poor performance, 3-6 was graded as acceptable, and $>6$ indicated good performance.

Results: For both analyzers, 2 levels IQC sigma values of chloride and sodium were $<3$, while the levels of alkaline phosphatase, aspartate aminotransferase, amylase, creatine kinase, iron, gamma-glutamyl transferase, and magnesium were $>6$; and the sigma values of total bilirubin, phosphorus, glucose, high-density lipoprotein-cholesterol, total cholesterol, calcium, creatinine, and total protein were determined to be within the acceptable range of 3-6. Amylase and creatine kinase were the best performers on both analyzers, while sodium had the lowest sigma values.

Conclusion: Six Sigma is a good method to evaluate the analytical process performance of a clinical laboratory. Quality control measures should be implemented for parameters with low sigma values.
\end{abstract}

Keywords: Laboratories, quality control, six sigma

C linical laboratory reports play a critical role in clinical decisions about patients. Therefore, clinical laboratories should evaluate process performance and minimize laboratory errors in order to produce the most accurate and reproducible test results possible. There are 3 basic stages in the total test process of medical laboratories: pre-analytical, analytical, post-analytical. It has been reported that $30 \%$ to $75 \%$ of laboratory errors occur in the pre-analytical phase, $9 \%$ to $55 \%$ in the post-analytical phase, and $4 \%$ to $30 \%$ in the analytical phase [1].

Laboratories should evaluate their process performance according to scientifically accepted quality criteria. This assessment includes the percentage of sample errors and rejections in the pre-analytical phase, the accuracy and precision measurement of test results in the analytical phase, and critical values reporting and test turnaround times in the post-analytic phase [2].

Clinical laboratories approve the validity of the analysis process according to quality control procedures for each analyte. Quality control consists of internal quality control (IQC) and external quality control (EQC) measures. IQC generally employs 2 or 3 levels of clinical decision points and daily IQC results are interpreted using control charts, such as the Levy-Jennings and Westgard rules. EQC samples are provided to clinical chemistry laboratories by an external agency once a month for use in analyzing and reporting [1].

Address for correspondence: Saniye Basak Oktay, MD. Department of Biochemistry, Adiyaman University Training and Research Hospital, Adiyaman, Turkey Phone: +90 5555540881 E-mail: snybasak@gmail.com ORCID: 0000-0002-3427-9893

Submitted Date: January 11, 2021 Accepted Date: March 12, 2021 Available Online Date: March 17, 2021

${ }^{\circ}$ Copyright 2021 by International Journal of Medical Biochemistry - Available online at www.internationalbiochemistry.com OPEN ACCESS This work is licensed under a Creative Commons Attribution-NonCommercial 4.0 International License. 
Analytical process errors comprise systematic and random errors that have basic parameters such as inaccuracy and imprecision. These parameters are expressed as bias and coefficient of variation (CV), respectively. Total error (TE) can be calculated using bias and $\mathrm{CV}$ for each test (TE=Bias+1.65CV) [3]. The allowable total error (TEa) is a parameter provided by reports such as the US Clinical Laboratory Implementation Amendments 1988 (CLIA'88) and the German RiliBÄK [4, 5]. Evaluation of the process performance of a clinical laboratory is essential for comparison with laboratories around the world and to ensure high quality standards. During the analytical phase, variables can be assessed according to quality control and calibration procedures [6]. Analytical process performance can be evaluated using process sigma levels, quality indicators, and patient test results [7].

Six Sigma is a quality management method that integrates accurate and precision evaluation, error identification, and process improvement. The Six Sigma method has been used in hospital quality management since 1999 [8]. The universal application steps are to define, measure, analyze, develop, and control. The sigma value can be calculated by laboratories using the TEa and bias and CV \% levels [sigma=(TEa \%-bias \%) /CV \%]. A higher sigma level reflects greater consistency and stability of laboratory tests. A low sigma value indicates poor quality, defined as defects per million opportunities (DPMO). The process sigma values according to DPMO recorded in this study are shown in Table 1 [9].

Bias and SD values, which are the criteria of accuracy and repeatability, are obtained from IQC or EQC programs regularly used in clinical laboratories. While some studies suggest calculating the bias values using IQC data, others recommend using EQC data [1, 10-12].

In this study, the analytical process performance of routine biochemical tests performed using 2 biochemistry analyzers in our laboratory was evaluated using Six Sigma methodology and compared.

\section{Materials and Methods}

The present study was conducted between January 1, 2020 and March 30, 2020 in the clinical chemistry laboratory of Adiyaman University Research and Education Hospital. The IQC data of 2 Abbott Architect c16000 analyzers (Abbott Diagnostics Inc., Lake Forest, IL, USA) were extracted for the following parameters: albumin (Alb), alkaline phosphatase (ALP), alanine aminotransferase (ALT), aspartate aminotransferase (AST), amylase (AMY), direct bilirubin (DBIL), total bilirubin (TBIL), creatine kinase (CK), iron (Fe), phosphorus (P), gamma-glutamyl transferase (GGT), glucose (Glc), high-density lipoprotein-cholesterol (HDL-Cholesterol), total cholesterol, calcium (Ca), chloride $(\mathrm{Cl})$, creatinine (CREA), lactate dehydrogenase (LD), lipase, magnesium $(\mathrm{Mg})$, potassium $(\mathrm{K})$, sodium (Na), total protein (TP), triglyceride (TG), uric acid (UA), and urea. The daily level 1 (normal concentration) and level 2 (abnormal concentration) IQC material used was Technopath
Table 1. Process sigma level according to defects per million opportunities

\begin{tabular}{lc} 
Sigma level & DPMO \\
\hline 1 & 691462 \\
2 & 308538 \\
3 & 66807 \\
4 & 6210 \\
5 & 233 \\
6 & 3.4 \\
\hline
\end{tabular}

DPMO: Defects per million opportunities.

MultiChem S Plus (Lot: 18609180; Technopath Life Sciences, Ballina, Co. Tipperary, Ireland) and was applied according to the manufacturer's instructions.

IQC level 1 ( $n=65$ for each test) and IQC level 2 ( $n=65$ for each test) data were obtained from the laboratory information system. The target mean values of the IQC material were those specified by the IQC material manufacturer and the target TEa levels were determined according to the CLIA'88. The CLIA total error criteria can be freely accessed at http://www.westgard.com.

The laboratory mean, SD, CV \%, bias \%, and sigma values were calculated for all of the analytes and the performance was graded according to the sigma level $(<3$ : poor performance, 3-6: acceptable, >6: good performance).

The statistical analysis was performed using Microsoft Office Excel 2007 (Microsoft Corp., Redmond, WA, USA). Non-acceptable control data were excluded from the study. The mean, SD, $\mathrm{CV} \%$, bias \%, and sigma values of the acceptable control data were calculated using the following the formulas:

$\mathrm{CV} \%=\mathrm{SD} /$ Mean $\times 100$

Bias \% $=($ Laboratory mean-Target Mean)/Target Mean $\times 100$ Sigma $=($ TEa-Bias $) / C V$

\section{Results}

The target mean, TEa level, laboratory mean, SD, CV \%, bias $\%$, and sigma values for IQC level 1 and level 2 of the tests run on the first Abbott Architect c16000 analyzer are shown in Table 2, and results for the second Abbott Architect c16000 analyzer are shown in Table 3 . Table 4 shows the distribution of analytes grouped according to the calculated sigma values.

The sigma values of Alb, DBIL, Cl, Na, urea, and TG for IQC level 1 and the sigma values of $\mathrm{Alb}, \mathrm{Cl}$, and $\mathrm{Na}$ for IQC level 2 were $<3$ on the first analyzer. The sigma values of $\mathrm{Cl}$ and $\mathrm{Na}$ for IQC level 1 , and the sigma values of Alb, DBIL, Cl, and Na for IQC level 2 were $<3$ on analyzer 2 .

The sigma values of ALT, TBIL, P, Glc, HDL-cholesterol, total cholesterol, Ca, CREA, TP, and UA for IQC level 1, and the sigma values of DBIL, TBIL, P, Glc, HDL-cholesterol, total cholesterol, 


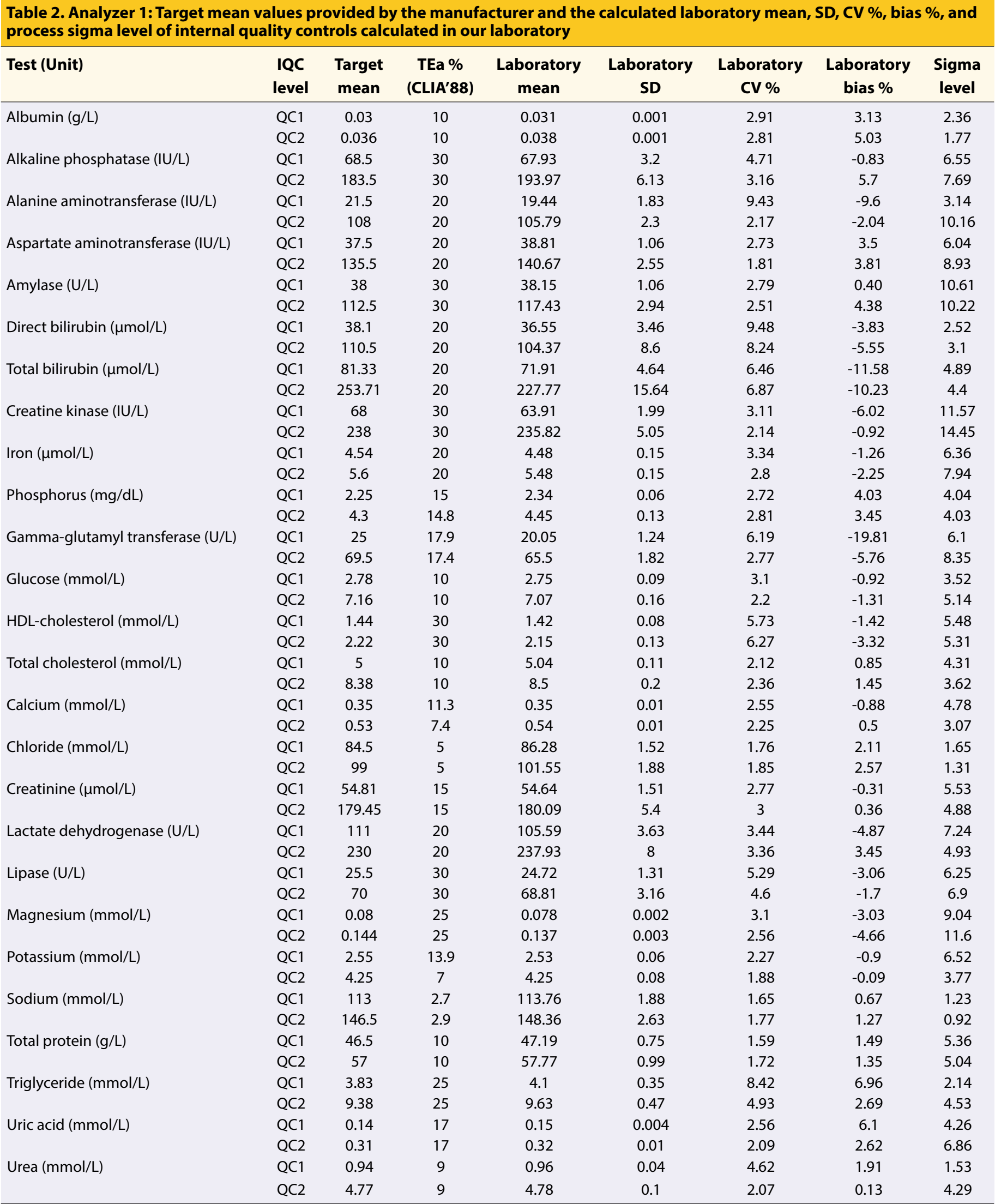

CLIA'88: Clinical Laboratory Implementation Amendments 1988; CV: Coefficient of variation; IQC: Internal quality control; TEa: Total allowable error; QC: Quality control; HDL: high-density lipoprotein. 


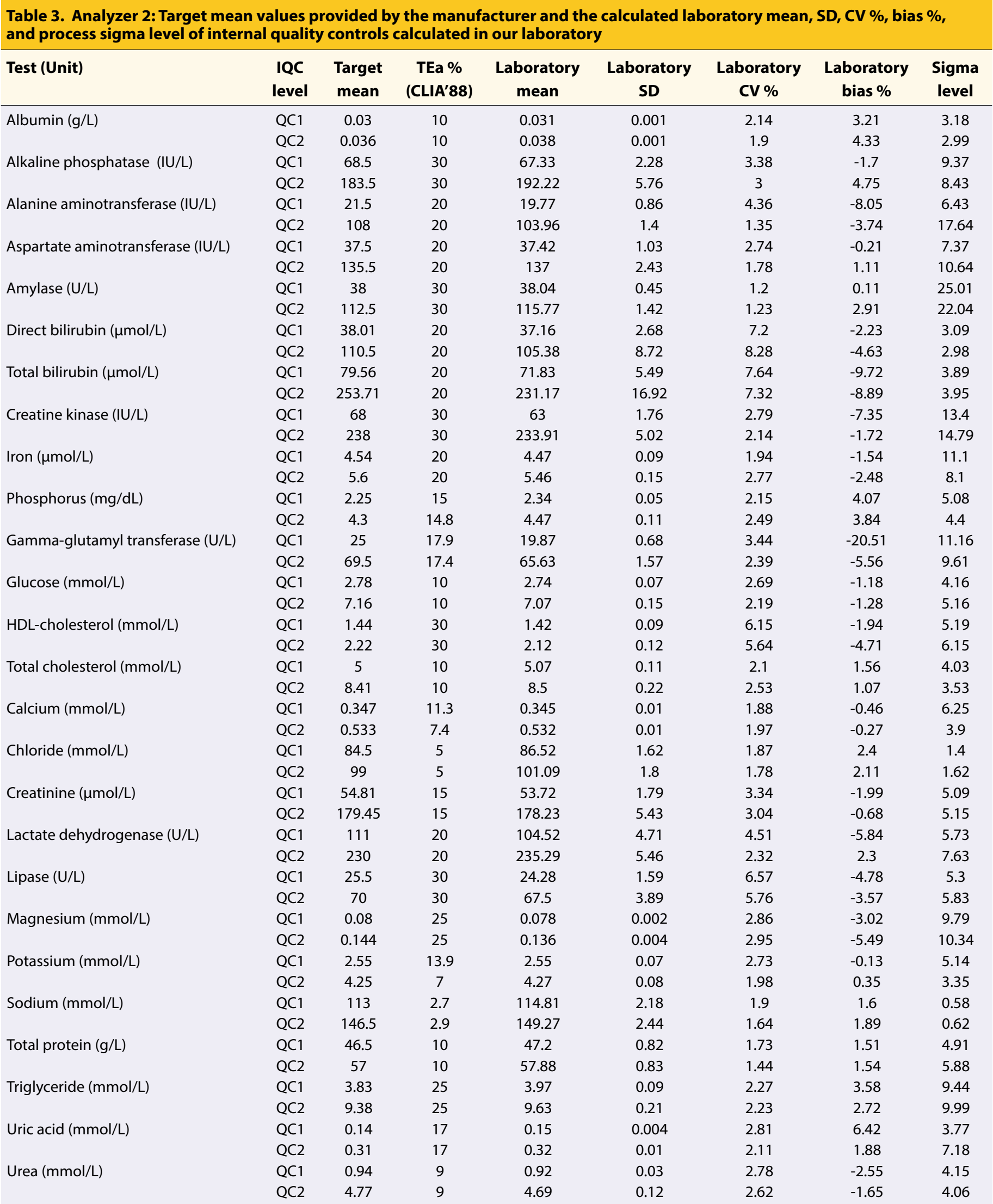

CLIA'88: Clinical Laboratory Implementation Amendments 1988; CV: Coefficient of variation; IQC: Internal quality control; TEa: Total allowable error; QC: Quality control; HDL: high-density lipoprotein. 
Table 4. Distribution of analytes grouped according to calculated sigma value

\begin{tabular}{|c|c|c|c|c|}
\hline \multirow[b]{2}{*}{ Sigma metrics } & \multicolumn{2}{|c|}{ Analyzer 1} & \multicolumn{2}{|c|}{ Analyzer 2} \\
\hline & QC1 & QC2 & QC1 & QC2 \\
\hline \multirow[t]{6}{*}{ Group 1 (<3 sigma) } & Albumin & Albumin & Chloride & Albumin \\
\hline & Direct bilirubin & Chloride & Sodium & Direct bilirubin \\
\hline & Chloride & Sodium & & Chloride \\
\hline & Sodium & & & Sodium \\
\hline & Urea & & & \\
\hline & Triglyceride & & & \\
\hline \multirow[t]{14}{*}{ Group 2 (3-6 sigma) } & ALT & Direct bilirubin & Albumin & Total bilirubin \\
\hline & Total bilirubin & Total bilirubin & Direct bilirubin & Phosphorus \\
\hline & Phosphorus & Phosphorus & Total bilirubin & Glucose \\
\hline & Glucose & Glucose & Phosphorus & Total cholesterol \\
\hline & HDL-cholesterol & HDL-cholesterol & Glucose & Calcium \\
\hline & Total cholesterol & Total cholesterol & HDL-cholesterol & Creatinine \\
\hline & Calcium & Calcium & Total cholesterol & Lipase \\
\hline & Creatinine & Creatinine & Creatinine & Potassium \\
\hline & Total protein & Lactate dehydrogenase & Lactate dehydrogenase & Total protein \\
\hline & Uric acid & Potassium & Lipase & Urea \\
\hline & & Total protein & Potassium & \\
\hline & & Triglyceride & Total protein & \\
\hline & & Urea & Uric acid & \\
\hline & & & Urea & \\
\hline \multirow[t]{12}{*}{ Group 3 (>6 sigma) } & Alkaline phosphatase & Alkaline phosphatase & Alkaline phosphatase & Alkaline phosphatase \\
\hline & AST & ALT & ALT & ALT \\
\hline & Amylase & AST & AST & AST \\
\hline & Creatine kinase & Amylase & Amylase & Amylase \\
\hline & Iron & Creatine kinase & Creatine kinase & Creatine kinase \\
\hline & GGT & Iron & Iron & Iron \\
\hline & Lactate dehydrogenase & GGT & GGT & GGT \\
\hline & Lipase & Lipase & Calcium & HDL-cholesterol \\
\hline & Magnesium & Magnesium & Magnesium & Lactate dehydrogenase \\
\hline & Potassium & Uric acid & Triglyceride & Magnesium \\
\hline & & & & Triglyceride \\
\hline & & & & Uric acid \\
\hline
\end{tabular}

QC: Quality control; ALT: Alanine aminotransferase; AST: Aspartate aminotransferase; GGT: Gamma-glutamyl transferase; HDL: High-density lipoprotein.

Ca, CREA, LD, K, TP, TG, and urea for IQC level 2 were within the acceptable range on analyzer 1 (sigma level: 3-6). For analyzer 2, the sigma values of Alb, DBIL, TBIL, P, GIc, HDL-cholesterol, total cholesterol, CREA, LD, lipase, K, TP, UA, and urea for IQC level 1, and the sigma values of TBIL, P, Glc, total cholesterol, Ca, CREA, lipase, K, TP, and urea for IQC level 2 were within the acceptable range (sigma level: 3-6).

ALP, ALT (except IQC level 1 on analyzer 1), AST, AMY, CK, Fe, lipase (analyzer 1), GGT, LD (except IQC level 2 on analyzer 1 and IQC level 1 on analyzer 2), Mg, TG (analyzer 2), UA (except IQC level 1 on both analyzers), K (IQC level 1 on analyzer 1 ), Ca (IQC level 1 on analyzer 2), and HDL-cholesterol (IQC level 2 on analyzer 2) had good performance (sigma level: $>6$ ).

\section{Discussion}

We evaluated 26 biochemical analytes using the Six Sigma methodology on 2 Abbott Architect c16000 analyzers and we compared IQC level sigma values. For both analyzers, the 2 levels of IQC sigma values of $\mathrm{Cl}, \mathrm{Na}$, and Alb (except IQC level 1 on analyzer 2) were <3; the 2 levels of IQC sigma values of ALP, AST, AMY, CK, Fe, GGT, Mg, TG (analyzer 2), ALT (except IQC level 1 on analyzer 1 ) were $>6$. The 2 levels of IQC sigma values of TBIL, P, Glc, HDL-cholesterol (except IQC level 2 on analyzer 2), total cholesterol, Ca (except IQC level 1 on analyzer 2), CREA, TP, and urea (except IQC level 1 on analyzer 1 ) were within the acceptable range (sigma level: 3-6). AMY and CK had the best 
sigma metrics on both analyzers, while $\mathrm{Na}$ had the lowest sigma values on both analyzers. The sigma values of many analytes were consistent for both analyzers.

Many studies have evaluated analytical performance using the Six Sigma method with different analyzers and parameters, and using internal or external quality controls. Medina et al. [12] evaluated 5 years of IQC data of 2 Abbott Architect $\mathrm{C} 8000$ chemistry analyzers. The sigma values of DBIL, CK, $\mathrm{HDL}$-cholesterol, TG, and UA were $>6$ for 1 analyzer while the values of $\mathrm{CK}, \mathrm{DBIL}, \mathrm{HDL}$-cholesterol, Mg, TG, and UA were $>6$ for the second analyzer. The electrolytes $\mathrm{Ca}, \mathrm{Cl}$, and $\mathrm{Na}$ had an average sigma level of $<3$ on both devices, while $\mathrm{K}$ showed better sigma scores.

Mao et al. [1] and Zhou et al. [13] extracted 5 months' worth of IQC data of biochemical parameters using the AU5800 analyzer (Beckman Coulter Inc., Brea, CA, USA). In the study performed by Mao et al. [1], the sigma values of urea and Na were determined to be $<3$; and AMY, UA, HDL-cholesterol, TBIL, ALT, TG, AST, ALP, and CREA were $>6$. Zhou et al. [13] reported that the sigma values of $\mathrm{BUN}, \mathrm{Ca}, \mathrm{ALT}$, and $\mathrm{P}$ were $<3$, and those of ALP, CK, TG, GGT, and TBIL were $>6$.

Other studies in the literature that used the Six Sigma method have yielded varied results [10,11,14-18]. The differences in sigma values of the analytes may be due to differences in the autoanalyzers, the quality control material, the pre/ post-analytical conditions, the period of study, or the method used.

The TEa target levels used to evaluate the analytical process influence the calculated sigma values. It has been noted that the different CLIA and RiliBÄKTEa levels affected the results [4, 5]. In our study, the TEa target values were determined according to the CLIA' 88 and the low sigma values of $\mathrm{Na}$ and $\mathrm{Cl}$ seen may have been due to the low TEa target levels used. A very stringent calibration, IQC, and analyzer maintenance have to be followed for parameters with low sigma values. Simple QC rules are adequate for parameters with high sigma values.

Six Sigma-method applications allow a laboratory to calculate their performance using universal criteria and to compare the results with those of other clinical laboratories around the world. Parameters with low performance can be identified using this analysis and performance should be improved with regulatory activities in order to meet the universal quality criteria. The sigma levels of $\mathrm{Na}, \mathrm{Cl}$, and Alb in our study indicated that regulatory activities should be conducted for low concentration and electrolyte tests that were studied with the indirect ion selective electrode method. Fluctuations in the electrolyte results may have been due to contamination or deterioration of the reference electrode. We implemented regulatory activities such as changing the reference solution and performing electrode maintenance more frequently to resolve these problems.

This study has some limitations that should be noted. First, the evaluation period was limited to 3 months. Second, because of the short duration, the EQC-Bias \% was not evaluated. We believe that EQC-Bias \% data calculated over longer periods would provide statistically more accurate results, and our next goal is to evaluate the EQC as well as the IQC over a longer period as a bias indicator.

In conclusion, the Six Sigma method is an effective form of statistical analysis to evaluate analytical process performance with quality control results. In the present study, the identification and measurement steps of the universal application were performed for biochemistry tests on 2 Abbott Architect c16000 analyzers. The next goal is to perform the analysis, improvement, and control steps to further enhance our analytical process performance.

Conflict of Interest: No conflict of interest is declared by the author.

Financial Disclosure: No financial disclosure is declared by the author.

Peer-review: Externally peer-reviewed.

Authorship Contributions: Concept - S.B.O., S.N.A.; Design S.B.O.; Supervision - S.B.O., S.N.A.; Funding - None; Materials S.B.O.; Data collection \&/or processing - S.B.O.; Analysis and/or interpretation - S.B.O., S.N.A.; Literature search - S.B.O., S.N.A.; Writing - S.B.O., S.N.A.; Critical review - S.B.O., S.N.A.

\section{References}

1. Mao X, Shao J, Zhang B, Wang Y. Evaluating analytical quality in clinical biochemistry laboratory using Six Sigma. Biochem Med (Zagreb) 2018;28(2):020904. [CrossRef]

2. Llopis MA, Trujillo G, Llovet MI, Tarrés E, Ibarz M, Biosca C, et al. Quality indicators and specifications for key analyticalextranalytical processes in the clinical laboratory. Five years' experience using the Six Sigma concept. Clin Chem Lab Med 2011;49(3):463-70. [CrossRef]

3. Westgard JO, Klee GG. Quality management. In: Burtis CA, Ashwood ER, Bruns DE, editors. Tietz textbook of clinical chemistry and molecular diagnostics. St. Louis, MO: Elsevier Saunders; 2006. p. 485-529.

4. RiliBÄK (Richtlinien der Bundesärztekammer) - guidelines of the German medical association for the quality assurance of laboratory medical examinations. Available at: http://www. westgard.com/rilibak.htm. Accessed Oct 30, 2017.

5. CLIA proficiency testing criteria. Available at: http://www. westgard.com/clia.htm. Accessed Oct 30, 2017.

6. Berte LM. Laboratory quality management: a roadmap. Clin Lab Med 2007;27(4):771-90, vi. [CrossRef]

7. Emekli Di. Tibbi laboratuvar akreditasyonunda toplam test süreci performansının değerlendirilmesi: altı sigma metodolojisi; 2012. Available at: http://acikerisim.pau.edu.tr:8080/ xmlui/handle/11499/2349. Accessed Mar 16, 2021.

8. Cano EL, Moguerza JM, Redchuk A. Six Sigma with R: statistical engineering for process Improvement. New York, NY: Springer Science + Business Media; 2012. [CrossRef]

9. Aslan $D$, Demir S. Laboratuvar tıbbında altı sigma kalite yönetimi. Turk J Biochem 2005;30:272-78. 
10. Nanda SK, Ray L. Quantitative application of sigma metrics in medical biochemistry. J Clin Diagn Res 2013;7(12):2689-91.

11. Ganji SB, Revupalli S. Evaluation of quality assurance in a new clinical chemistry laboratory by six sigma metrics. J Clin Diagn Res 2019;13(3):BC04-BC07. [CrossRef]

12. Medina PA, Matibag J, Datay-Lim SJ, Arcellana-Nuqui E. A Pilot Study on the Evaluation of Clinical Chemistry Laboratory Test Performance using Six Sigma Metrics. PJP 2019;4(2):31-36. [CrossRef]

13. Zhou B, Wu Y, He H, Li C, Tan L, Cao Y. Practical application of six sigma management in analytical biochemistry processes in clinical settings. J Clin Lab Anal 2020;34(1):e23126. [CrossRef]

14. Singh B, Goswami B, Gupta VK, Chawla R, Mallika V. Application of sigma metrics for the assessment of quality assurance in clinical biochemistry laboratory in India: a pilot study. Indian J Clin Biochem 2011;26(2):131-35. [CrossRef]

15. Kumar BV, Mohan T. Sigma metrics as a tool for evaluating the performance of IQC in a clinical chemistry laboratory. J Lab Physicians 2018;10(2):194-99. [CrossRef]

16. Nar R, Emekli DI. The evaluation of analytical performance of Immunoassay tests by using six-sigma method. J Med Biochem 2017;36(4):301-8. [CrossRef]

17. Maksane SN, Parikh R, Vaswani L. Quantitative Assessment of Analytical Phase Quality of Clinical Biochemistry Parameters Using Sigma Metrics. IJML 2017;4(2):81-90.

18. Emekli Di, Güçlü K, Turna G, İlanbey B, Nar R, Çiçek EA. Klinik laboratuvarlarda analitik süreç değerlendirilmesi: altı sigma metodolojisi. Türk Klinik Biyokimya Derg 2016;14(2):79-86. 